\title{
Experimental Investigation of Wide Bandwidth Single and Dual Pump non-Degenerate Phase Sensitive Amplifiers
}

\author{
Joseph Kakande*, Francesca Parmigiani, Morten Ibsen, Periklis Petropoulos \\ and David J. Richardson \\ Optoelectronics Research Centre, University of Southampton, Southampton, SO17 1BJ, UK \\ *jkk@orc.soton.ac.uk
}

\begin{abstract}
We demonstrate non-degenerate phase sensitive amplification of a DPSK data signal in both single and double pumped parametric configurations, achieving uniform phase sensitive gain and attenuation over the $20 \mathrm{~nm}$ assessed bandwidth.

(C)2010 Optical Society of America

OCIS codes: (060.2320) Fiber optics amplifiers and oscillators, (190.4410) Nonlinear optics, parametric processes
\end{abstract}

\section{Introduction}

Phase sensitive amplifiers (PSAs) have long attracted considerable interest due to their ability to perform low noise amplification below the $3 \mathrm{~dB}$ phase insensitive quantum limit [1]. They have the potential to be applied to all-optical signal processing, including phase and amplitude regeneration of high speed phase encoded signals [2-3]. In [4], we experimentally demonstrated the precise control of continuous wave $(\mathrm{CW})$ signals in a single pump parametric amplifier to achieve non-degenerate PSA, revealing practical limits to achieving the theoretical maximal phase sensitive (PS) attenuation, particularly as signal (idler) gain and/or detuning in wavelength from the pump increases. In this paper, we further investigate these limitations and demonstrate flat, broadband and spectral distortion free phase sensitive gain of 40Gbit/s differential phase shift keying (DPSK) signals in a single pumped non-degenerate PSA operating in the low gain regime. Additionally, we consider two pump phase sensitive amplifiers (2P-PSA) which have the benefits, compared to the single pump configuration, of avoiding idler spectral broadening due to pump modulation if the pumps are counter phase modulated, as well as requiring lower peak pump powers reducing susceptibility to stimulated Brillouin scattering (SBS). We demonstrate for the first time to our knowledge a wideband non-degenerate 2P-PSA.

\section{Experimental Setup and Results}

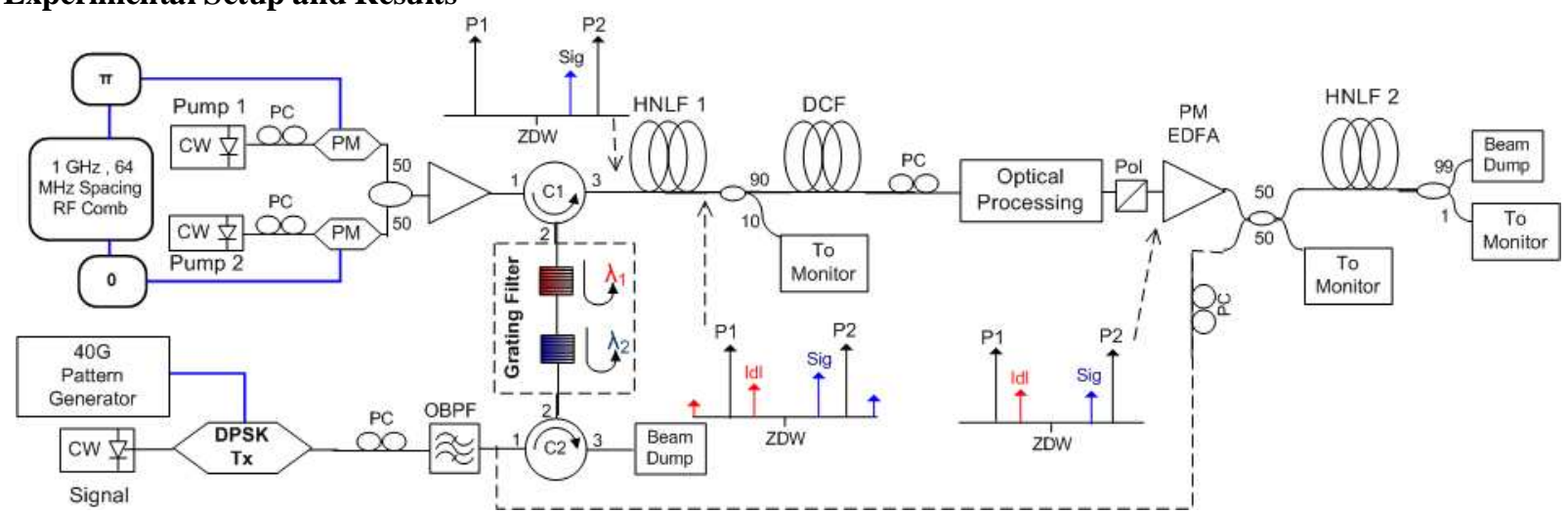

Figure 1: Phase sensitive amplifier setup, $\mathrm{CW}-$ tunable laser source, $\mathrm{PC}-$ polarisation controller, $\mathrm{PM}$ - phase modulator, $\mathrm{Pol}-$ Polariser, OBPF - optical bandpass filter.

The experimental setup is shown in Fig. 1. Two tunable lasers were counter-phase modulated [5] using a 1GHz RF comb of $64 \mathrm{MHz}$ spaced lines to suppress SBS before being combined in a $3 \mathrm{~dB}$ coupler and amplified to provide pumps at 1540 and $1560.5 \mathrm{~nm}$. Alternatively, one of the lasers was turned off and the other tuned to $1550 \mathrm{~nm}$ to convert to the single pump equivalent. The pump(s) were then launched into port 1 of circulator $\mathrm{C} 1$ and reflected off a single (dual) window $0.5 \mathrm{~nm}$ full width at half maximum (FWHM) fiber grating filter to reject any out-of-band amplified stimulated emission (ASE). A third tunable laser was externally modulated to provide a 40 Gbit/s DPSK signal that was coupled into port 1 of circulator $\mathrm{C} 2$. Since the signal wavelength was different as compared to the pump(s), it was transmitted through the grating and, thus, coupled together with the pump(s) emerging from circulator $\mathrm{C} 1$ port 3 with a pump insertion loss $<1 \mathrm{~dB}$. The pump(s) and signal were launched into a highly nonlinear 
fiber, HNLF 1, to generate an idler, which was phase locked to the signal and pump(s). The HNLF was $500 \mathrm{~m}$ long with a nonlinear coefficient of $11 / \mathrm{W} / \mathrm{km}$ and a zero-dispersion wavelength (ZDW) of $1555 \mathrm{~nm}$ (OFS Fitel, Denmark). This was followed by a few meters of dispersion compensating fiber (DCF) to compensate for the dispersion between HNLFs 1 and 2, an optical processor (Finisar Waveshaper 4000E) to control the signals' relative amplitude and phase (as required to achieve PSA operation), a polarizer to ensure uniform polarization alignment and a polarization maintaining erbium doped fiber amplifier (PM-EDFA) to boost the signals up to $0.5 \mathrm{~W}$ with the pump(s) dominating the power. The amplified waves were then coupled into HNLF 2 which was $220 \mathrm{~m}$ long with a nonlinear coefficient of 11/W/km, and ZDW of $1550 \mathrm{~nm}$ via a $3 \mathrm{~dB}$ coupler where it experienced PS amplification. The second port of the coupler was used to launch the data signal from the transmitter directly into HNLF 2 to allow measurements of the absolute phase insensitive amplification (PIA) by comparing the HNLF2 output on an optical spectrum analyzer (OSA) with the pump on and off, respectively.

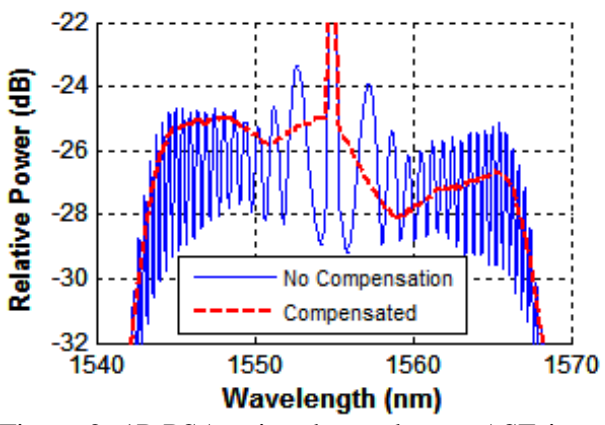

Figure 2: 1P-PSA gain when coherent ASE is coupled into the PSA. Solid curve - no dispersion pre-compensation, dotted curve - compensated.

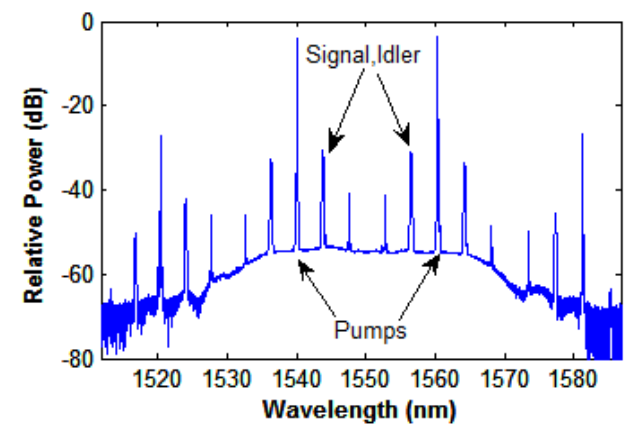

Figure 4: 2P-PSA output spectra showing unwanted broadband secondary mixing products, when the signals relative phase is optimized for maximum gain.

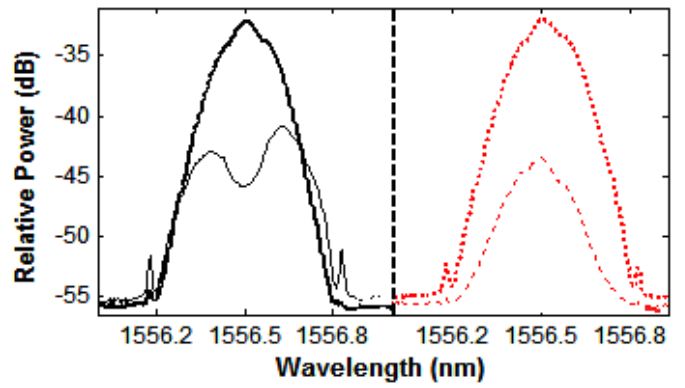

Figure 3: Spectral traces of a $40 \mathrm{Gbit} / \mathrm{s}$ DPSK signal at 2PPSA output. Black solid thick (thin) line - No compensation PS maximum (minimum), red dashed thick (thin) line - compensated PS maximum (minimum).

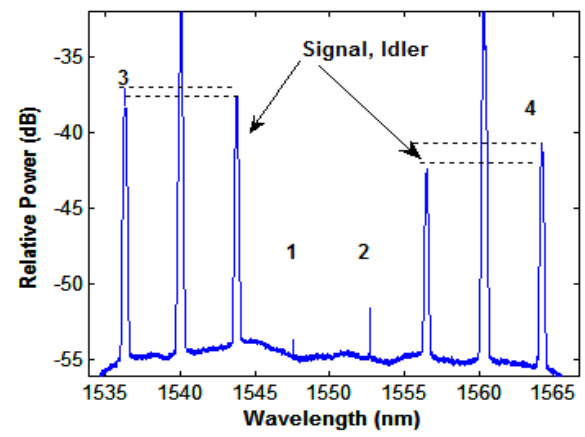

Figure 5: Impact of second order mixing products on $2 \mathrm{P}-$ PSA minimum attenuation spectra; secondary mixing products labelled 1-4.

The 1P-PSA gain when coherent ASE from the PIA is coupled into the PSA is shown in Fig. 2. The solid curve shows the characteristic gain ripple when the dispersive elements prior to the input of the PSA (HNLF2) are not properly compensated, causing a wavelength dependent relative phase modulation [6]. The dotted curve shows a close to optimally chromatic dispersion compensated case (via optimization of the DCF length). It appears asymmetric mainly due to having coupled asymmetric ASE spectrum into the PSA. Note that similar curves are obtained with the 2P-PSA. Figure 3 shows the impact of dispersion compensation on the non-degenerate PSA of a $40 \mathrm{Gbit} / \mathrm{s}$ DPSK signal in the considered 2P-PSA. From these optical spectra, it is clear that amounts of dispersion corresponding to just a few metres of SMF prior to the PSA can greatly distort the transmitted data signals, especially in the case of maximum attenuation, due to the PSA phase transfer function [4]. It is also observed that similar values of minimum attenuation are achieved at the very centre of the spectrum for both compensated and uncompensated systems, implying that dispersion affects wideband but not narrowband PSA behaviour.

Figure 4 shows an optical spectral measurement for a 2P-PSA. As compared to 1P-PSA, a major limitation of the 2P-PSA in a low dispersion slope HNLF is the generation of secondary mixing products which act to deplete the pumps, see Fig. 4. In addition, the presence of extra sidebands due to the coexistence of single pump interactions with the signals at the PSA input also affects the PS operation, particularly during PS attenuation. This can be seen 
in Fig. 5 for the case of maximal attenuation - the sidebands labelled 3 and 4 are generated at an early stage in HNLF 2, experience PIA and eventually become larger than the signals, and start to affect the amount of signal attenuation achievable, adding noise to the signals in the process. To overcome this issue, we limited ourselves to low-gain operation of the PSA, which is outlined below.

Figures 6 and 7 show the PS maximal gain, maximal attenuation and PIA gain achieved for 1P and 2P-PSA. The PIA gains in both cases are around $1 \mathrm{~dB}$, with the maximal PS gain being 4 to $5.5 \mathrm{~dB}$ higher. The 1P-PSA gain is flat due to the pump proximity to the fibre ZDW. In the 2P-PSA case the gain drops towards the centre of the bandwidth but this is to be expected based on the average pump wavelength being slightly higher than the ZDW (the selection of wavelengths was primarily limited by the grating filters that were available for our experiments). Note that the maximal gain and attenuation are equal and opposite, as expected theoretically over the full $20 \mathrm{~nm}$ operating bandwidth assessed, in accordance with theory [6]. This was possible in part due to the use of a PM-EDFA which ensured that the waves are always co-polarised at the PSA input. Achieving a large PS attenuation is fundamentally more difficult than a large PS gain, but is crucial in order to achieve both good phase regeneration and a noise figure approaching $0 \mathrm{~dB}$, two of the most intriguing promises of PSA.

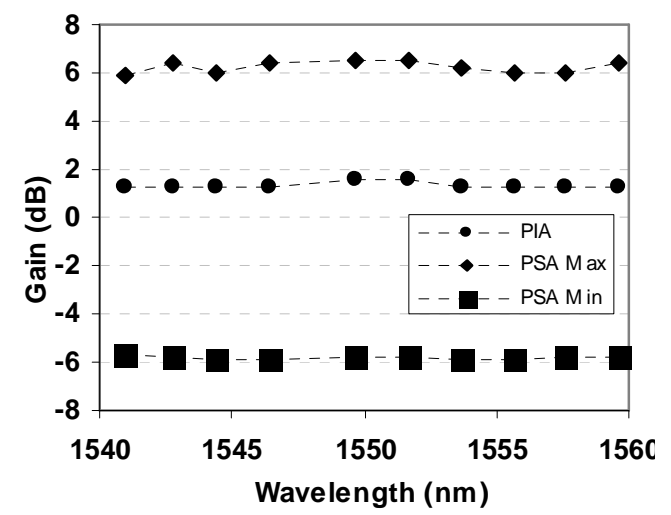

Figure 6: 1P-PSA gain over 20nm for a $0.5 \mathrm{~W}$ pump at $1550 \mathrm{~nm}$.

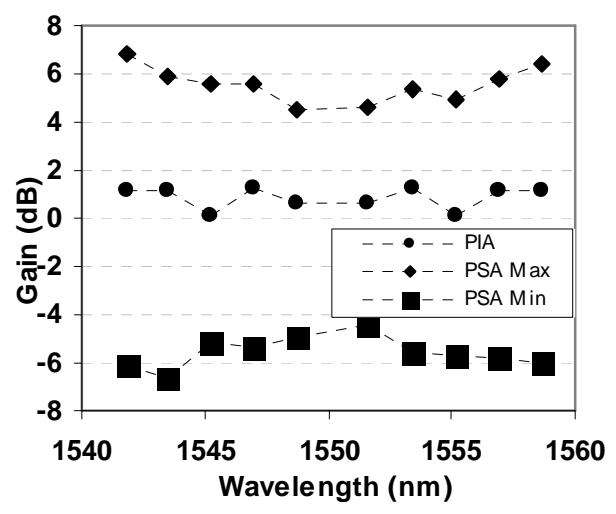

Figure 7: 2P-PSA gain over $20 \mathrm{~nm}$ for pumps at 1540 and $1560.5 \mathrm{~nm}$ and a total power of $0.5 \mathrm{~W}$.

Note that far greater gains can be achieved both for the $1 \mathrm{P}$ and $2 \mathrm{P}$ cases but only with a compromise both to the gain flatness and stability of maximal attenuation, for example, as observed in Ref [4] for the 1P-PSA case. However it is to be appreciated that in most cases of interest, e.g. PSAs for phase processing and regeneration, high maximal PSA gains are not the greatest priority since the regeneration capability is determined mainly by the difference between the maximal gain and attenuation.

\section{Conclusions}

We have compared one- and two-pump non-degenerate phase sensitive amplification of wideband signals in FOPA, including the first demonstration to our knowledge of a dual-pump non-degenerate PSA. By compensating for the signals dispersion and polarization rotation before the PSA, we were able to achieve flat gain and attenuation of $40 \mathrm{Gbit} / \mathrm{s}$ signals over a 20 -nm bandwidth without any dispersion induced spectral distortion. Equal and opposite values of gain and attenuation are achieved by operating at low PSA gains.

This research has received funding from the European Communities Seventh Framework Programme FP/2007-2013 under grant agreement 224547 (PHASORS)

\section{References}

[1] H. A. Haus and J. A. Mullen, "Quantum Noise in Linear Amplifiers," Physical Review 128, 2407 (1962)

[2] K. Croussore, I. Kim, Y. Han, C. Kim, G. Li, and S. Radic, "Demonstration of phase-regeneration of DPSK signals based on phasesensitive amplification," Opt. Express, 13, 3945-3950 (2005).

[3] K. Croussore and G. Li, “Amplitude regeneration of RZ-DPSK signals based on four-wave mixing in fibre,” Electron. Lett. 43, 177-178 (2007)

[4] C. Lundström, et al., "Experimental Comparison of Gain and Saturation Characteristics of a Parametric Amplifier in Phase-sensitive and Phase-insensitive Mode," in Proceedings of European Conference on Optical Communications, Vienna, Austria 2009

[5] A. A. Vedadi, et al., "Demonstration of an Integrated LiNbO3 Synchronized Double Phase Modulator and Its Application to Dual-Pump Fiber Optical Parametric Amplifiers and Wavelength Converters," J. Lightwave Technol. 26, 777-781 (2008).

[6] R. Tang, J. Lasri, P. S. Devgan, V. Grigoryan, P. Kumar, and M. Vasilyev, "Gain characteristics of a frequency nondegenerate phasesensitive fiber-optic parametric amplifier with phase self-stabilized input," Opt. Express 13, 10483-10493 (2005) 